\title{
Pinning of the martensitic microstructures by dislocations in $\mathrm{Cu}_{74.08} \mathrm{Al}_{23.13} \mathrm{Be}_{2.79}$
}

\author{
H. Idrissi ${ }^{1}$, D. Schryvers ${ }^{\text {a1 }}$, E.K.H. Salje ${ }^{2}$, H. Zhang ${ }^{2}$, M.A. Carpenter ${ }^{2}$, X. Moya ${ }^{3}$ \\ ${ }^{1}$ EMAT, University of Antwerp, Groenenborgerlaan 171, B-2020 Antwerp, Belgium \\ 2 Department of Earth Sciences, University of Cambridge, Downing Street, Cambridge CB2 3EQ, UK \\ ${ }^{3}$ Department of Material Sciences, University of Cambridge, Pembroke Street, Cambridge CB2 3QZ, UK
}

\begin{abstract}
A single crystal of $\mathrm{Cu}_{74.08} \mathrm{Al}_{23.13} \mathrm{Be}_{2.79}$ undergoes a martensitic phase transition at $246 \mathrm{~K}$ and $232 \mathrm{~K}$ under heating and cooling, respectively. Surprisingly, the martensite phase is elastically much harder than the austenite phase showing that interfaces between various crystallographic variants are strongly pinned and can not be moved by external stress while the phase boundary between the austenite and martensite regions in the sample remains mobile. This unusual behavior was revealed by Dynamical Mechanical Analysis and Resonant Ultrasound Spectroscopy. Transmission Electron Microscopy shows that the pinning is generated by dislocations, which are inherited from the austenite phase. Such dislocations can hinder the movement of stacking faults in the $18 \mathrm{R}$ martensite structure or twin boundaries between martensite variants.
\end{abstract}

\section{Introduction}

The overall observation in most martensitic systems is that the product phase is elastically much softer than the austenitic phase because some, or all, internal interfaces between the martensite variants move under external stress [1,2]. However, it was observed that the untrained ternary $\mathrm{Cu}_{74.08} \mathrm{Al}_{23.13} \mathrm{Be}_{2.79}$ alloy displays a very different behavior. Instead of softening in the martensite phase the material becomes effectively harder. This was concluded from measurements from dynamical mechanical analysis (DMA) at low frequencies and resonant ultrasonic spectroscopy (RUS) at frequencies in the $\mathrm{kHz}$ region [3]. Fig. 1 shows the temperature dependence of the elastic constants $\mathrm{C}_{44}$ and $1 / 2\left(\mathrm{C}_{11}-\mathrm{C}_{12}\right)$ obtained from RUS experiments. The change in the elastic constants when crossing the transition temperatures around $240 \mathrm{~K}$ clearly reveals this increase in hardness in the martensite. This could either mean that martensite-martensite interfaces do not exist or that such interfaces are heavily pinned. In order to investigate an uncommon increase in hardness upon transforming to martensite, the material was investigated by conventional two-beam and in-situ cooling transmission electron microscopy (TEM). More detail on the experiments and used instruments will be given in a forthcoming paper [3].

\section{Results and discussion}

Most samples from the original rod used for the DMA, RUS and differential scanning calorimetry (DSC) measurements did not reveal any martensite at room temperature, which corresponds with the transformation temperatures below room temperature (RT). Also in-situ cooling by liquid nitrogen did not result in large martensite plates due to thin foil effects of the electron transparent regions. Fortunately, a small composition difference at the end of the rod provided a sample with transition temperatures around RT in which some $18 \mathrm{R}$ martensite plates could be observed at RT, as seen in the BF two-beam image of Fig. 2. The 18R structure of the martensite is clear from the row of superlattice reflections in the [-2-10] selected area electron diffraction (SAED) pattern shown in the inset and originating from the encircled region inside the martensite plate.

a e-mail: nick.schryvers@ua.ac.be

This is an Open Access article distributed under the terms of the Creative Commons Attribution-Noncommercial License (http://creativecommons.org/licenses/by-nc/3.0/), which permits unrestricted use, distribution, and reproduction in any noncommercial medium, provided the original work is properly cited. 
In Fig. 2 a high density of dislocations in the austenite matrix regions can be observed. Some of these dislocations, in particular those with dislocation lines close to perpendicular to the trace of the austenitemartensite interface, are seen to propagate from the austenite into the martensite plate trough the austenitemartensite interface. These dislocations will consequently affect the plasticity of the martensite. A second family reveals dislocation lines close to parallel to the trace of this particular austenite-martensite interface.

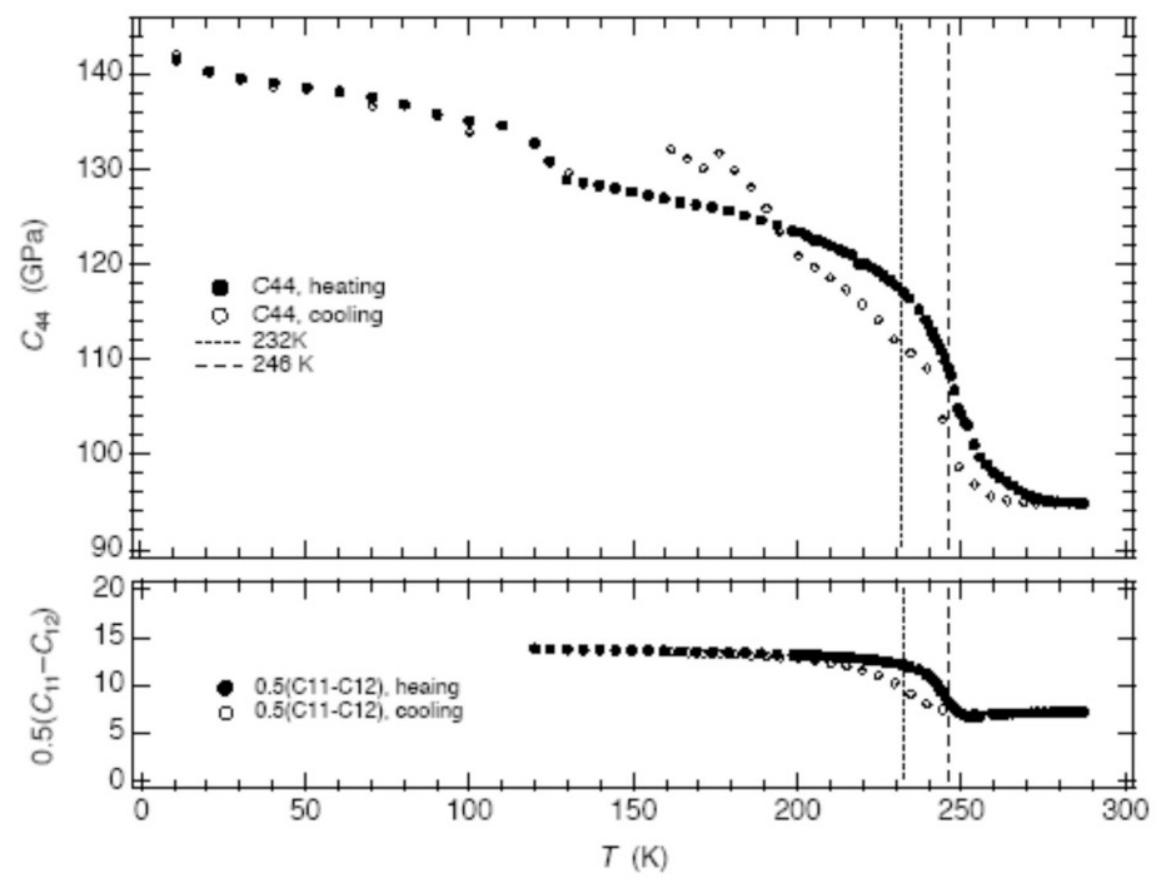

Fig. 1. Variations in elastic constants obtained from fitting to resonance peaks in RUS measurements with frequencies of $\sim 284 \mathrm{kHz}$ (a) and $\sim 65 \mathrm{kHz}$ (b) at room temperature. Broken lines represent transition temperatures taken from a DSC analysis. The hardening in the martensitic phase is clearly visible.

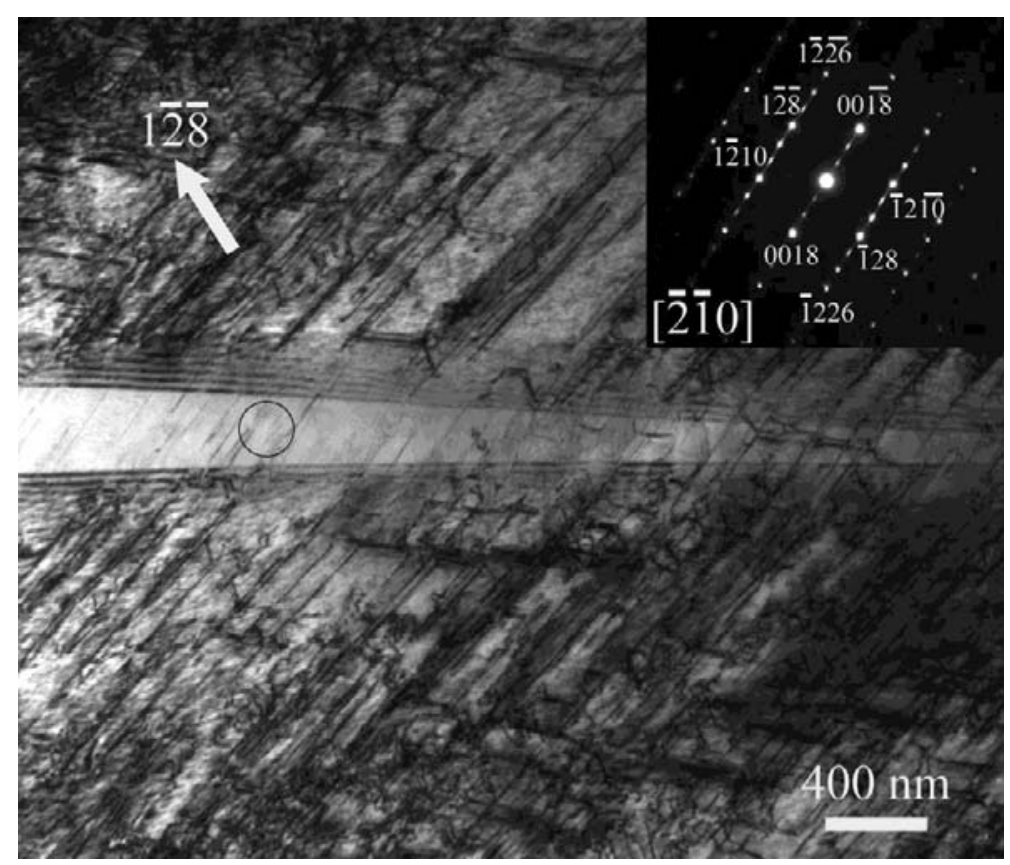

Fig. 2. Two-beam BF micrograph showing a martensite plate inside the austenite matrix revealing an abundance of dislocations, close to perpendicular to and some close to parallel with the trace of the austenite-martensite interface. The inset shows a SAED of the 18R martensite originating from the encircled region. 
Fig. 3a is a weak beam dark field (WBDF) image obtained using the 0018 reflection corresponding with the basal or close packed planes of the martensite. This image again reveals contrast of two populations of dislocations, types $\mathrm{A}$ and $\mathrm{B}$, but now inside the martensite plate. It can be expected that the glide planes for populations A and B correspond to (-128) and (0018) planes of the martensite structure, respectively, since the dislocation lines are parallel to these planes. In addition, an intersection between an A and a B dislocation is indicated by a black arrow. At the intersection point, the deviation of the line orientation of the B type dislocation is seen. The origin of these dislocations can be deduced by carefully analyzing the image of Fig. 3a. Dislocations of type A in the martensite are seen to originate from the austenite at spots marked by white arrows. The small orientation deviation exhibited by these dislocations when crossing the interface is probably due to the orientation difference between the glide planes in both phases. Also, although no type B dislocations are seen to cross the austenite-martensite interface, Fig. 2 clearly shows a family of dislocations in the austenite again parallel with the martensite type B ones. In other words, it is assumed that also the observed type B dislocations in the martensite originate from the latter family of dislocations in the austenite.

In Fig. 3b, the close packed stacking of the $18 \mathrm{R}$ structure becomes visible as a result of the imaging contrast of many parallel stacking defects. Halfway the plate, and indicated by a white arrow, some of these stacking defects are seen to stop at a dislocation running close to parallel with the long axis of the martensite plate. This indicates that these dislocations in the martensite and inherited from the austenite, have a clear impact on stacking defects of the $18 \mathrm{R}$ martensite structure.
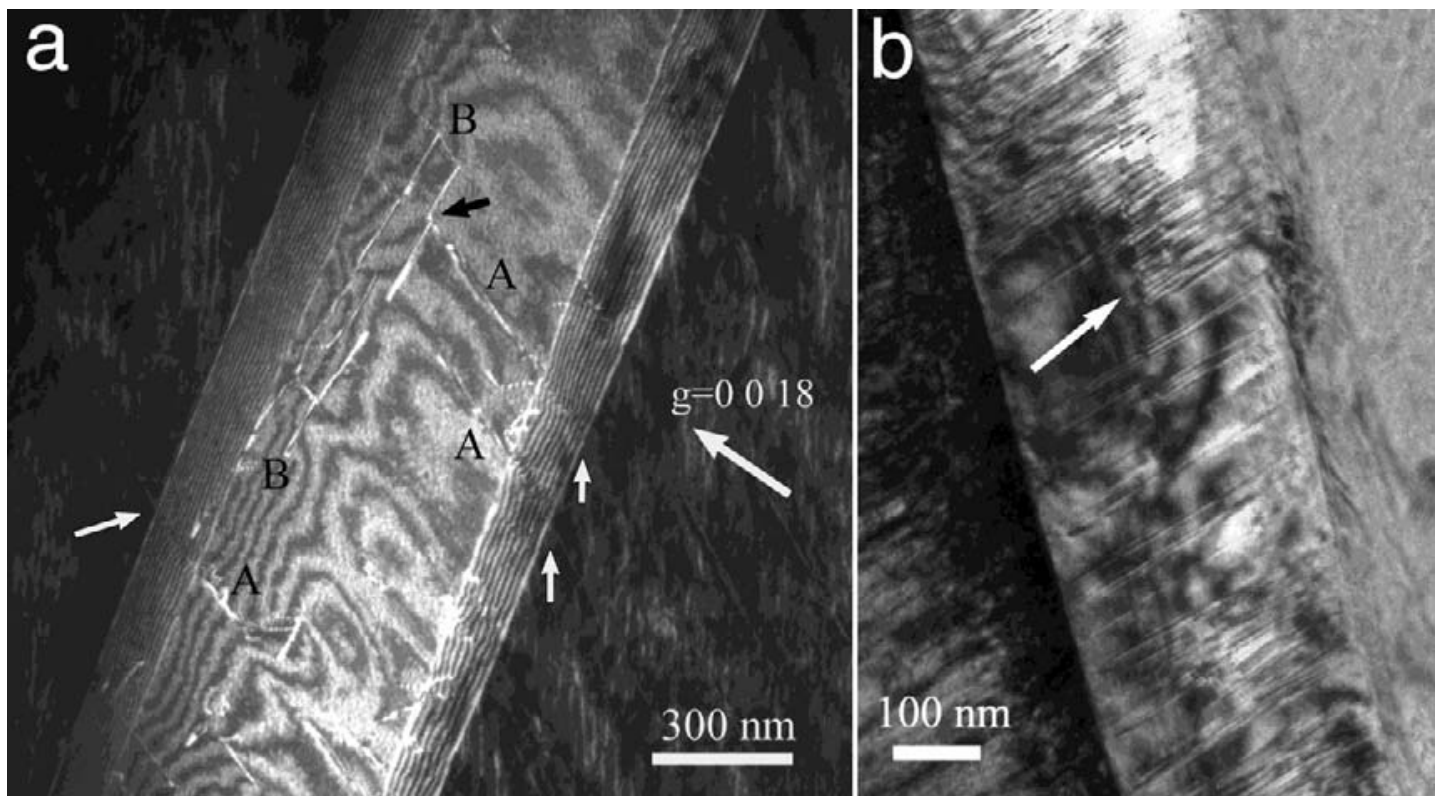

Fig. 3. (a) WBDF micrograph showing both types of martensite dislocations A and B inherited from the austenite. (b) Stacking defects of the $18 \mathrm{R}$ stacking are seen to stop at a dislocation inside a martensite plate (white arrow).

By tilting away the sample from the [-2-10] zone axis it could be concluded that the type A dislocations in the martensite correspond to a couple of partial dislocations (PD) bounding stacking faults (SF) with a dissociation of around $15 \mathrm{~nm}$ [3]. Stacking faults on $\{12-8\}$ type planes were observed before in $18 \mathrm{R}$ martensite of $\mathrm{Cu}-\mathrm{Al}-\mathrm{Ni}$ alloys $[4,5]$. Recently, screw dislocations on the (-128) plane and which do not extend into partials were reported by Zhang et al. [6] in a deformed 18R martensite $\mathrm{Cu}-\mathrm{Zn}$-Al shape memory alloy.

For the dislocations of type B, which are parallel to the (0018) glide plane, it can be expected that they also correspond to two PD bounding a SF, since the contrast of these dislocations in Fig. 3a shows discontinuous features (also observed for type A dislocations). This phenomenon is due to the superposition of the contrast of the partial dislocations and the SF fringes when the SF plane is parallel to the electron beam. In the literature, such dislocations gliding in the $(001)_{18 \mathrm{R}}$ plane were reported by Rodriguez et al. [7,8] in plastically deformed 18R martensite of $\mathrm{Cu}-\mathrm{Zn}-\mathrm{Al}$ alloys.

In very few cases the martensite plates revealed the expected twinning as seen in Fig. 4a, where the twin character is clear from the orientation of the stacking on both sides of the midrib as well as from the spot splitting in the SAED inset. The reason for the low fraction of twinning is probably the fact that the martensitic transition has been captured in a very early stage so that the total driving force for introducing twins as stressrelief mechanism is not yet very large. In Fig. $4 \mathrm{~b}$ the same region has been oriented in such a way as to visualize dislocation lines in one of both martensite variants and reaching the midrib or twin interface as indicated by white arrows. 

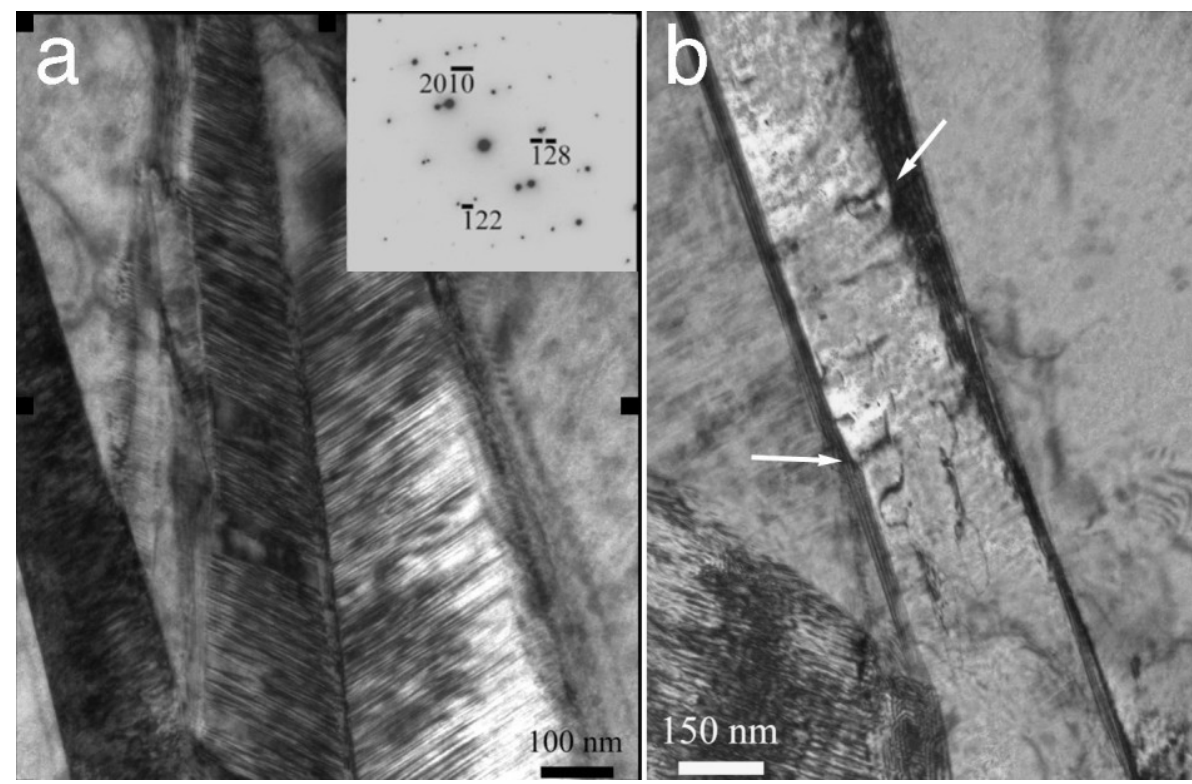

Fig. 4. (a) martensite plate showing a twin midrib with the corresponding SAED as inset revealing the respective spot splitting. (b) dislocation lines arriving at the twin midrib (left) and habit plane (right) of the martensite plate.

In order to study the influence of the dislocations on the hardness of the martensite, an in-situ cooling experiment was carried out. Fig. 5 shows a two-beam DF image obtained in the martensite at $-20^{\circ} \mathrm{C}$. In this image, the nearly vertical bright lines correspond to the previously characterized type A dislocations. In the same image, close to horizontal bright lines parallel to the trace of the $18 \mathrm{R}$ c-plane can also clearly be seen. During the in-situ cooling experiment, dislocations of type A or B actively crossing the austenite-martensite interface were not observed. On the other hand, the horizontal bright lines were rapidly crossing the viewing screen inside the martensite plate. It can thus be concluded that these horizontal bright lines are intrinsic to the martensite. These new defects probably correspond to partial dislocations originating from the imperfections of the $18 \mathrm{R}$ stacking and are dragging large SF behind them, such as the defects already noticed in Fig. $3 \mathrm{~b}$. The small arrows indicate some intersection points between these expected PD and the earlier described type A dislocations. At the intersection point the brightness increases due to a local increase of the strain at the intersection point. In some cases, the moving partial dislocation is even seen to stop at the type A dislocation, which corresponds to the location indicated by the white arrow in the still image of Fig. $3 \mathrm{~b}$.

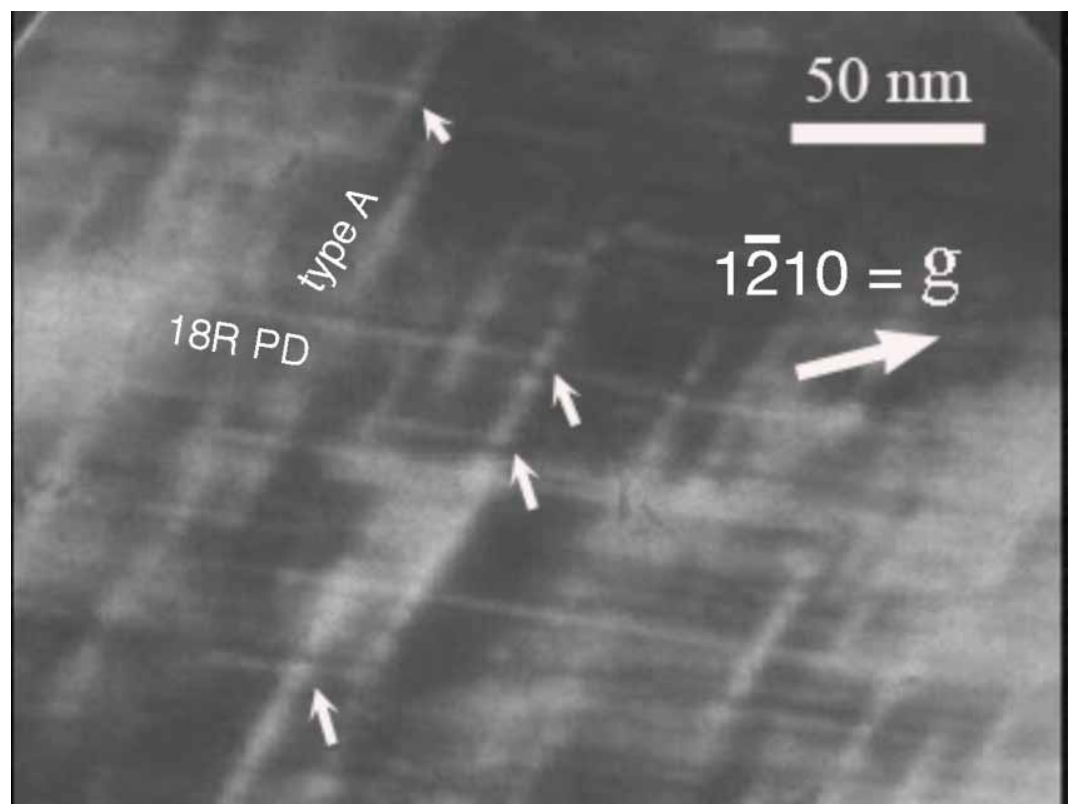

Fig. 5. Two-beam DF micrograph obtained at $-20^{\circ} \mathrm{C}$ showing an enhanced strain contrast at white arrows where transformation dislocations (close to horizontal white lines) tangle with type A dislocations (close to vertical white lines). 
The above observations thus indicate that, during the transformation, PD bounding SF will rapidly pass through the product phase on different close packed planes. The driving force for the SF expansion is a combination of the local stress in the martensite plate and the phase transformation, which tends to produce a new and energetically more stable structure. The PD at the tip of the SF will be pinned when intersecting a dislocation inherited from the austenite. Although the result of the dislocation interaction depends on the geometry of the interaction and dislocation type, we can expect that the pinning effect of the extended dislocations of type A will be more important compared to a perfect dislocation due to the elastic strain field of the two dissociated partial dislocations at the type A dislocation and the additional stress at the SF in between. For the present plate, type A dislocations will be the most potent pinning sites due to the particular orientation of the martensite plate and its internal long period stacking, but for other plates or variants oriented in different ways, other families of dislocations could take over this prominent role.

Since no moving twin interfaces could be observed, essentially due to experimental limitations such as limited range of tilt, thin film effect hampering the martensitic transformation and the fact that only very few martensite plates were observed in the first place, an actual live interaction between dislocations and twin interfaces could not be captured. Nevertheless, since the movement of a twin interface occurs in the same way as that of a single SF, it can readily be assumed that the same type of dislocation interaction can pin the twin planes in the same way as they do the 18R stacking faults. Obviously, the above observation performed in a cooling experiment is typical of what is expected to occur when the sample is under load or mechanical properties are measured. In other words, also under those circumstances, the same dislocation pinning processes are expected to hamper the 18R stacking faults and twin interfaces to move as they should in an elastically soft martensite.

\section{Conclusions}

The elastic response in $\mathrm{Cu}_{74.08} \mathrm{Al}_{23.13} \mathrm{Be}_{2.79}$ is unusual in so far as only the interface between the austenite and the martensite phase are somewhat mobile while the interfaces between the martensite variants are immobile. TEM images of an untrained austenite phase show a multitude of dislocations along different crystallographic planes. This is unusual for untreated shape memory alloys such as the Ni-Ti systems. These dislocations are inherited by the martensite phase during the transformation. For some families of dislocations and depending on the orientation of a particular martensite plate or variant, the orientation of the dislocation line is nearly perpendicular to the basal plane of the $18 \mathrm{R}$ structure. Additional basal plane partial dislocations appear during the transformation. These additional partial dislocations dominate the microstructure of the advancing martensite phase. Twin boundaries as expected for the accommodation of the martensite variants at low temperatures would move by the same basal plane dislocation mechanisms of the $18 \mathrm{R}$ stacking. This movement we find pinned by crossing dislocations. Kustov et al. [9] used the term of 'dislocation jamming' of the interface mobility when they analyzed the effect of plastic deformation and heat treatment on the athermal stabilization of the martensite phase. In our experiments the interface mobility between austenite and martensite is still not suppressed while the jamming mechanism appears to relate strongly to the twin boundaries between the martensitic variants.

Support from Marie-Curie RTN MULTIMAT (contract no. MRTN-CT-2004-505226) is acknowledged. DS and HI thank R. Espinoza for support with the TEM sample preparations. The sample was kindly provided by A. Planes from the Departament d'Estructura i Constituents de la Matèria of the Universitat de Barcelona. XM acknowledges support from Comissionat per a Universitats i Recerca (CUR) del Departament d'Innovacio, Universitats I Empresa, de la Generalitat de Catalunya.

\section{References}

[1] J. Van Humbeeck, J. Alloys Compd. 355, 58 (2003)

[2] Y.N. Wang, X.H. Chen, H.M. Shen, Chin. J. Met. Sci. Technol. 7, 157 (1991)

[3] E.K.H. Salje, H. Zhang, H. Idrissi, D. Schryvers, M.A. Carpenter, X. Moya, A. Planes, Phys. Rev. B, submitted (2009); E.K.H. Salje, H. Zhang, this meeting

[4] K. Otsuka, T. Nakamura, K. Shimizu, Trans. Japan. Inst. Metals 17, 200 (1974)

[5] J.W. Brooks, Electron Microscopy 1, 178 (1975)

[6] J.X. Zhang, Y.F. Zheng, Y.C. Luo, L.C. Zhao, Acta mater A 47, 3497 (1999)

[7] P.L. Rodriguez, A. Cuniberti, R. Romero, F.C. Lovey, Scripta Metall. et Mater. 27, 1133 (1992)

[8] P.L. Rodriguez, A.M. Condo, F.C. Lovey, Phys. Stat. Sol (b) 197, 279 (1996)

[9] A. Kustov, J. Pons, E. Cesari, M. Morin, J. Van Humbeeck, Materials Science and Engineering A 373, 328

(2004) 